Abb. 1 Überwiegend pflanzenbasierte Kostformen wie die mediterrane Vollwertkost haben sich in wissenschaftlichen Untersuchungen und in der Praxis als günstig zur Gewichtsreduktion erwiesen. @ karandaev/Adobe Stock
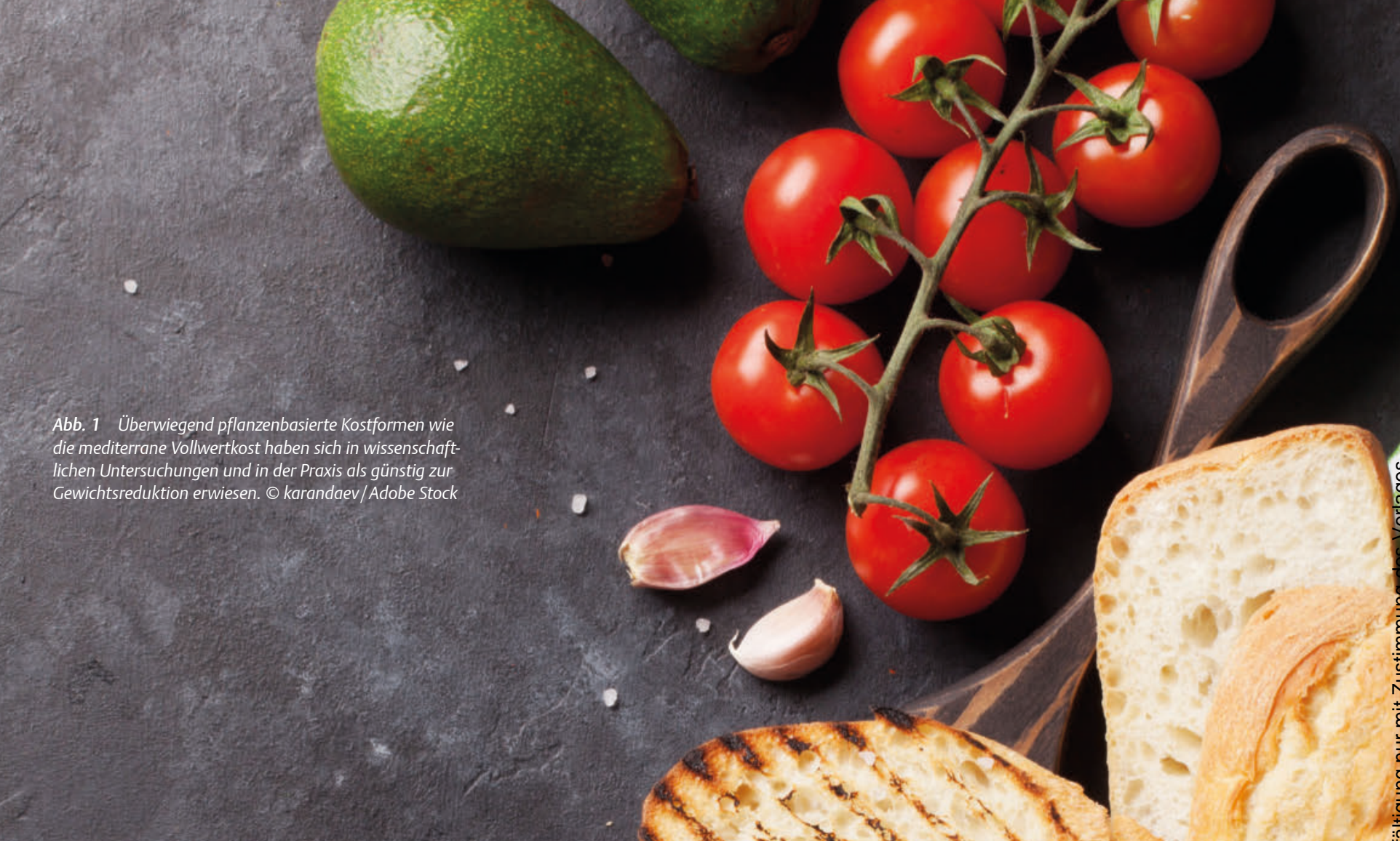

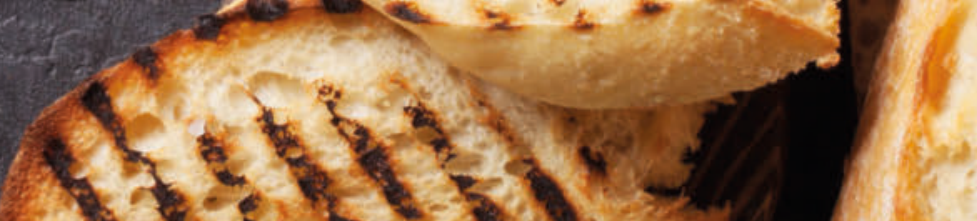

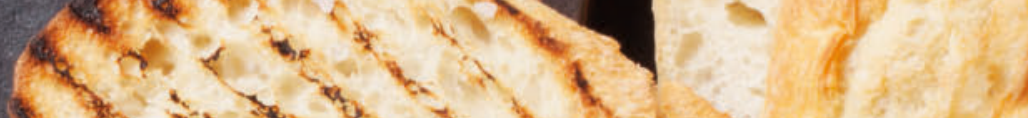

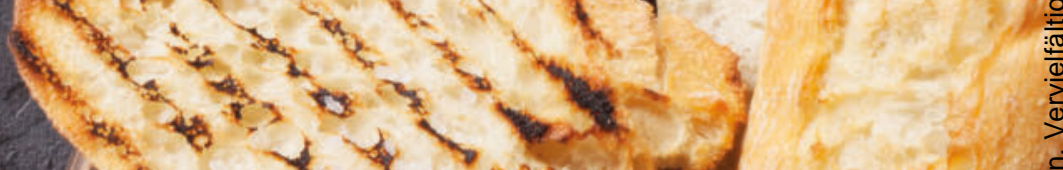

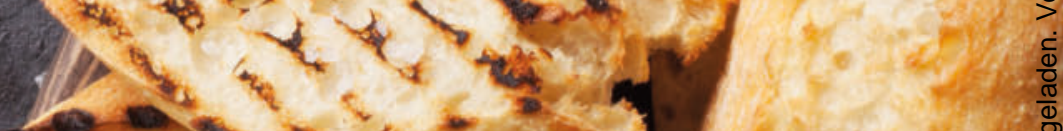

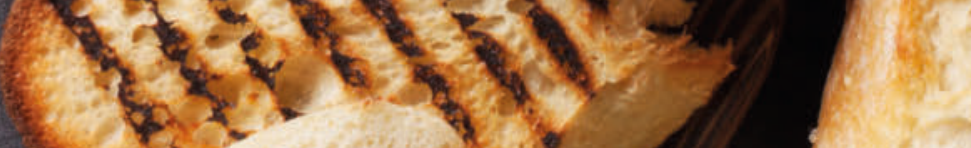

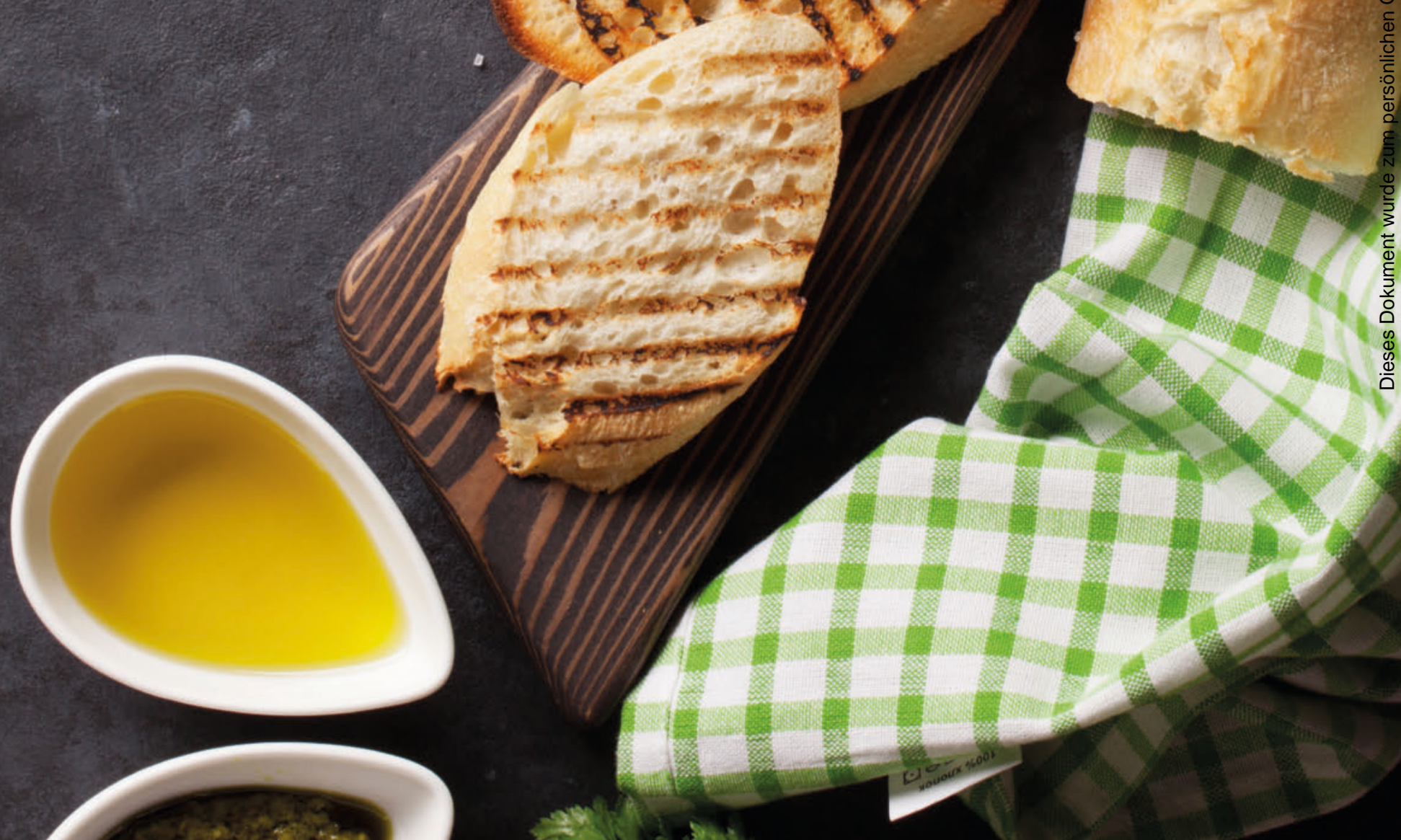




\title{
Naturheilkunde und
}

Komplementärmedizin bei

Herz-Kreislauf-Erkrankungen

Teil 1: Bluthochdruck

\author{
Gewichtsreduktion, Bewegung, Stressreduktion - Lebensstilfaktoren zu modifizieren \\ gehört zu den effektivsten Interventionen bei der Blutdrucksenkung \\ Andreas Michalsen, Rainer Stange
}

Herz-Kreislauf-Erkrankungen stellen unverändert die häufigste Todesursache der Bevölkerungen industrialisierter Regionen dar. Insbesondere durch die rapide Zunahme des metabolischen Syndroms und des Bluthochdrucks in den Schwellenländern wächst der Therapie- und Präventionsbedarf für HerzKreislauf-Erkrankungen weltweit. Parallel haben sich den letzten 20 Jahren erhebliche technisch-interventionelle, pharmakologische sowie kardio- und angiochirurgische Fortschritte und Entwicklungen ergeben. Allerdings sind diese Verfahren v.a. in der Akutversorgung erfolgreich, wohingegen sie in der Behandlung chronischer kardiovaskulärer Erkrankungen meist palliativen Charakter haben.

Vor diesem Hintergrund kommt den Verfahren der Naturheilkunde und Komplementärmedizin eine wachsende Bedeutung

\section{Zusammenfassung}

Am klinischen Zentrum für Naturheilkunde in Berlin werden kardiovaskuläre Erkrankungen seit Jahren erfolgreich mit einem multimodalen Konzept behandelt. Schwerpunkte bilden die Ernährungs- und Fastentherapie, Phyto-, Ordnungs-, Hydro-, Thermo- und Bewegungstherapie sowie ausleitende Verfahren.

Der Artikel gibt einen Überblick zu den auf Erfahrungen beruhenden Empfehlungen sowie Studienresultaten naturheilkundlicher und integrativer Therapiemöglichkeiten bei Hypertonie. zu. Am klinischen Zentrum für Naturheilkunde in Berlin werden kardiovaskuläre Erkrankungen seit Jahren erfolgreich mit einem multimodalen Konzept behandelt. Schwerpunkt sind hierbei die Ernährungsund Fastentherapie, die Phytotherapie, die Ordnungstherapie bzw. Mind-Body Medizin, die Hydro- und Thermotherapie, die Bewegungstherapie sowie ausleitende Verfahren. Im Folgenden werden die therapeutischen Möglichkeiten bei Hypertonie vorgestellt. Teil 2 (ab Seite 18) thematisiert die Behandlungsoptionen bei koronarer Herzkrankheit und Herzinsuffizienz.

\section{Bluthochdruck - arterielle Hyper- tonie}

Die mangelnde Therapietreue (Compliance) ist ein vorrangiges Therapieproblem in der Bluthochdrucktherapie. Nach einem Jahr hören 30-50\% der Patienten auf, ein verschriebenes Antihypertensivum einzunehmen. Dies unterstreicht die Bedeutung nicht-pharmakologischer Therapieverfahren zur Erreichung des Zielblutdrucks von mindestens $<140 / 90 \mathrm{mmHg}$ oder 138/85 mmHg. Die grundsätzlichen Empfehlungen zur Änderung des Lebensstils sind inzwischen Bestandteil internationaler Leitlinien, werden aber in der konventionellen Praxis nur selten ausreichend umgesetzt.

\section{Lebensstilmodifikation Gewichtsreduktion und Gewichts- normalisierung}

Ziel ist das Erreichen eines normalen Körpergewichtes mit einem BMI unter $25 \mathrm{~kg} / \mathrm{m}^{2}$ oder zumindest das Verhindern einer weiteren Gewichtszunahme. Bei Frauen sollte der Bauchumfang unter $80 \mathrm{~cm}$, bei Männern unter $94 \mathrm{~cm}$ sein. Bei $10 \mathrm{~kg}$ Gewichtsabnahme ist eine systolische Blutdrucksenkung von 5 bis zu $20 \mathrm{mmHg}$ möglich. Zum Erreichen dieses Ziels ist die Ernährungstherapie vorrangig.

\section{Ernährungstherapie}

In der antihypertensiven Therapie haben sich folgende Kostformen wissenschaftlich wie praktisch bewährt:

Pflanzenbasierte Kost bzw. vegetarische Ernährungsformen

Zahlreiche Studien haben die Wirksamkeit vegetarischer und v.a. veganer, rein pflanzenbasierter Kostformen zur Absenkung eines erhöhten Blutdrucks belegt. Einige pflanzliche Nahrungsmittel haben spezifisch blutdrucksenkende Wirkung (s.u.). Wichtig ist, auf eine vollwertige vegetarische bzw. vegane Ernährung zu achten bzw. „Puddingvegetarismus“ zu vermeiden. Für die vegane Ernährung ist unbedingt auf eine Vitamin- $\mathrm{B}_{12}$-Supplementierung zu achten.

Mediterrane Vollwertkost

Diese Ernährungsform ist zu ihrem überwiegenden Anteil ebenfalls pflanzenbasiert. 
Käse und Joghurt werden regional typisch in kleinen Mengen verzehrt. In küstennahen Regionen wurde traditionell auch etwas Fisch verzehrt. Hier ist heute aber insbesondere aus ökologischen Gründen der Verzehr von pflanzlichen $\omega$-3-Fettsäuren (z.B. $\alpha$-Linolensäure insbesondere in Leinsamen) oder von Algen bzw. Algenprodukten, die langkettige marine $\omega$-3-Fettsäuren enthalten, zu empfehlen. Zusätzlich hat sich die reichliche Verwendung von Olivenöl und Nüssen als vorteilhaft erwiesen.

\section{Laktovegetabile und pflanzenbasierte Vollwerternährung nach Leitzmann}

Hier stehen v. a die Prinzipien der Vollwertigkeit auch im ökologischen und sozialen Kontext sowie die Einbeziehung hoher Frischkostanteile im Vordergrund.

\section{DASH-Diät}

Im amerikanischen Raum hat sich die DASH-Diät (Dietary Approaches to Stop Hypertension) etabliert. Diese besteht primär aus einer salz- und fettreduzierten Ernährung mit hohen Anteilen von Frischgemüse und Obst. Systolische Blutdrucksenkungen von $8-14 \mathrm{mmHg}$ sind beschrieben. Entscheidender Unterschied zu den mediterranen bzw. vegetarischen Kostformen ist die Verwendung von fettreduzierten Milchprodukten und geringen Mengen Fleisch. Allen Ernährungsformen gemeinsam sind die hohen Gemüse- und Obstanteile und damit der Kaliumreichtum, der allein blutdrucksenkend wirkt, verstärkt durch moderate Kochsalzreduktion.

\section{Kochsalzreduktion}

Der allgemeine Nutzen einer Kochsalzreduktion ist trotz immer wieder aufflackernder Kontroversen erwiesen. Hierbei ist dem Patienten weniger eine vorgegebene Zahl (etwa max. 6g/d Kochsalz) mitzuteilen, sondern zu empfehlen, keinen Salzstreuer am Tisch und wenig meist stark gesalzenes „Convenience Food“ (Tiefkühl-, Fertiggerichte), oder „Fast Food“ zu verzehren. Hauptbeiträge in der durchschnittlichen hiesigen Ernährung stammen weiterhin aus zu stark gesalzenem konventionellen Brot sowie Fleisch, Wurst, Käse und insbesondere ihren Sekundärprodukten.

\section{Kempner'sche Reisdiät}

Der deutsch-amerikanische Nephrologe Walter Kempner entwickelte die Reisdiät

\title{
Heilfasten - bereits nach 24 Stunden sinkt der Blutdruck durch die initial gesteigerte Natriurese
}

\author{
erheblich ab.
}

und konnte damit vor der Ära der medikamentösen Blutdrucktherapie auch schwerste Fälle von therapieresistentem Bluthochdruck erfolgreich behandeln. Verzehrt werden bei dieser Diät über mehrere Wochen täglich mehrere große Portionen Reis bis zur Sättigung, ungesalzen und mit geringen Mengen Obstbrei.

\section{Mäßigung des Alkoholkonsums}

Die täglich zugeführte Menge an Alkohol sollte bei Männern nicht mehr als $30 \mathrm{~g}$, bei Frauen höchstens $15 \mathrm{~g}$ betragen. Hierdurch ist eine Senkung des systolischen Blutdrucks um 2-4 mmHg zu erwarten. Durch Abstinenz kann bei $90 \%$ der Patienten mit einer alkoholbedingten Hypertonie der Blutdruck normalisiert werden.

\section{Nahrungsergänzungen}

Im Bereich der Nahrungsergänzungen und spezifischen Nahrungsmittel sind als blutdrucksenkend (jeweils systolisch) einzuordnen:

- Leinsamen (die Wirksamkeit ist in placebokontrollierten Studien belegt)

- grüner Tee (Blutdrucksenkung 3-5 mmHg, mehr als 3 Tassen/d)

- nitrathaltige grüne Blattgemüse und rote Bete (Blutdrucksenkung bis zu $12 \mathrm{mmHg}$, ca. 0,51 Saft/d)

- Walnüsse (Blutdrucksenkung 2-3 mmHg, 50g/d)

- dunkle Schokolade (Blutdrucksenkung bis $3 \mathrm{mmHg}$, mind. $10 \mathrm{~g} / \mathrm{d}$ )

- Sojaeiweiß (z. B. Tofu und Tempeh; Blutdrucksenkung bis $9 \mathrm{mmHg}$, definierte Menge unklar)

- Kalium und Magnesium: Die blutdrucksenkende Wirkung beider Mikronährstoffe ist seit langem bekannt. Idealerweise werden Magnesium- und Kaliumzufuhr durch die Ernährung sichergestellt. Ggf. können Behandlungsversuche mit Supplementen erfolgen, Magnesium 400-800 mg/ $\mathrm{d}$ und Kalium 100-500 mg/d.

- Pistazien

- Heidelbeeren

\section{Probiotika}

Nach den Ergebnissen einer Metaanalyse haben Probiotika eine leichte blutdrucksenkende Wirkung zwischen 2 und $5 \mathrm{mmHg}$ systolisch. Unklar ist allerdings, welche Probiotika hier am besten wirken. Bei entsprechenden Hinweisen auf Verdauungsprobleme bzw. Vorliegen eines Reizdarmsyndroms ist eine probatorische Therapie mit einem gängigen Probiotikum oder eine betont präbiotische Ernährung (Inulin, Wurzelgemüse, Quinoa u.a.) zu empfehlen.

\section{Heilfasten, therapeutisches Fasten und modifiziertes Fasten}

Bei Bluthochdruck sehr bewährt und in Studien dokumentiert ist die Wirksamkeit des therapeutischen Fastens oder Heilfastens. Bereits nach 24 Stunden sinkt der Blutdruck durch die initial gesteigerte Natriurese erheblich ab. Antihypertensive Medikamente sind beim Fasten niedriger zu dosieren. Eine diuretische Therapie ist unter Kontrolle abzusetzen, da sonst gegebenenfalls gefährliche Hyponatriämien auftreten. Bei längerem Fasten treten weitere antihypertensiv wirksame Effekte ein, so die vegetative Umstimmung, Gewichts- und Fettmassenreduktion sowie Sensibilisierung von blutdruckregulierenden Rezeptorsystemen (v.a. natriuretische Peptide). Insgesamt sinkt der Blutdruck während eines längeren Fastens meist mehr als 20/10 mmHg ab.

Nach Kostaufbau steigen die Blutdruckwerte häufig wieder leicht an, bleiben jedoch unter dem Ausgangsniveau mit dann entsprechend reduziertem Medikamentenbedarf für eine begrenzte Zeit. Neben den wiederholt dokumentierten antihypertensiven Effekten zeigt Heilfasten günstige Wirkungen auf die nachhaltige Ernährungsumstellung. Eine Fastentherapie sollte i.d.R. unter klinischer Überwachung erfolgen. Die Fastendauer ist abhängig vom Ausgangsgewicht und der Konstitution. In der Regel wird eine 7- bis 14-tägige Fastentherapie durchgeführt.

Nicht zu empfehlen sind Nulldiäten aufgrund des stärkeren Proteinkatabolismus und des gesteigerten Risikos für Jo-Jo-Effekte. 


\section{Naturheilkunde und Komplementärmedizin Praxis zkm}

Bestehen Kontraindikationen für eine Fastentherapie sind oftmals Entlastungstage in Form von Obst- oder Reistagen eine für den Patienten gut praktikable Lösung. Entlastungstage wirken i.d.R. deutlich entwässernd und blutdrucksenkend, Reistage (Reistage: $150 \mathrm{~g}$ Vollkornreis ungesalzen, zusätzlich 750-1000 g Obst; $1000 \mathrm{kcal}$ ) sind modifiziert als Kempner'sche Diät bereits früher in der Bluthochdrucktherapie etabliert.

\section{Intervallfasten}

In den letzten 2-3 Jahren hat das Intervallfasten große Popularität erlangt (s. zkm 2/2018: 10-15). Nachdem sich in Tierversuchen wiederholt eine blutdrucksenkende und herzschützende Wirkung durch intermittierendes Fasten gezeigt hatte, sind in den letzten Jahren v.a. klinische Studien zum 5:2-Fasten (5 Tage normale Ernährung, 2 Tage Fasten in ständiger Fortführung), zum alternierenden Fasten (1:1) und zum „time restricted feeding“ (TRF) mit 16 Stunden Fasten und 8 Stunden Essen durchgeführt worden. Bei allen diesen Formen kommt es durch eine summarische Reduktion der Kalorienzufuhr von etwa $10 \%$ zu einer Gewichtsabnahme, aber auch zusätzlich zu blutdrucksenkenden Effekten. Auch in der eigenen Erfahrung erscheint das Intervallfasten als eine empfehlenswerte und sehr gut verträgliche ergänzende Therapiemethode bei Bluthochdruck.

\section{Bewegungstherapie}

Der blutdrucksenkende Effekt ist mehrfach nachgewiesen. Eine gute Wirksamkeit ist insbesondere bei vorher immobilen Patienten zu erwarten. Eine täglich oder mindestens 3-mal wöchentlich 30 min lang ausgeübte Aktivität senkt den systolischen Blutdruck um 4-9 mmHg und reduziert das kardiovaskuläre Risiko. Zu empfehlen sind pro Woche 4- bis 5-malige Ausdaueraktivitäten von 30-45 min. Die Art der Aktivität (z.B. Walking, Joggen, Radfahren, Schwimmen, Ergometertraining) sollte den individuellen Fähigkeiten und Neigungen angepasst werden, damit sie praktikabel ist und langfristig beibehalten wird. Vorzuziehen sind Aktivitäten mit geringem Verletzungsrisiko und geringer Wetterabhängigkeit. Joggen ist wegen kumulativer Verletzungsgefahr nur bedingt ratsam. Auch eine Fragmentierung der Bewegung in kleinere Einheiten ist wirksam, z. B. dreimal $10 \mathrm{~min}$ statt einmal 30 min Walking.

\section{Kombinierter Effekt von Lebens- stilmodifikationen}

Die kombinierte Anwendung der aufgeführten Lebensstilmodule führt nicht zu einer komplett additiven systolischen Blutdrucksenkung, sonst wären 40-50 mmHg denkbar. Zwar erreicht die nicht-pharmakologische Therapie mit den einzelnen Komponenten jeweils eine verlässliche Blutdrucksenkung, sind jedoch bereits einige Maßnahmen durchgeführt worden, wird der Effekt zusätzlicher Lebensstilmaßnahmen geringer. Dies bedeutet jedoch keine Einschränkung der Bedeutung der Lebensstiltherapie in der Bluthochdrucktherapie. Vielmehr ist dies ein Hinweis darauf, dass nicht alle Lebensstilmodule zur Maximierung der Blutdrucksenkung komplett erfüllt sein müssen, um deren Potenzial und konsekutiv die Notwendigkeit bzw. Intensität einer weiteren medikamentösen Therapie abzuschätzen.

\section{Ordnungstherapie und Mind-Body-Medizin}

Psychosoziale Faktoren und Stress sind gesicherte bedeutsame Risikofaktoren für die Entstehung der KHK und des Bluthochdrucks. Fehlender sozialer und emotionaler Rückhalt, Feindseligkeit, Angst, Depression, Arbeitsbelastungen können das Krankheitsbild verschlechtern und zu therapieresistentem Bluthochdruck beitragen. Unter dem Begriff „essenzielle Hypertonie“ verbergen sich fast immer Risikokonstellationen mit hohem Disstress, der sekundär einen ungünstigen Lebensstil verstärkt. In der Bluthochdrucktherapie können einzelne Entspannungsverfahren, im Rahmen komplexerer Programme der Mind-Body-Therapie wie in Tageskliniken zum Einsatz kommen.

Für die folgenden einzelnen Verfahren ist eine antihypertensive Wirksamkeit nachgewiesen.

- Meditation und Achtsamkeitstraining (Wirkung durch Metaanalysen belegt)

- Yoga (in Metaanalysen belegt)

- Tai-Chi und Qigong (durch einzelne hochwertige Studien belegt)

- Progressive Muskelrelaxation nach Jacobson (PMR) und Autogenes Training

Hierbei ist Meditation vermutlich anderen Verfahren überlegen. Blutdrucksenkungen von ca. $-10 \mathrm{mmHg}$ systolisch sind in kontrollierten Studien belegt. Nach kontrollierten Studien ist bei $75 \%$ der Patienten mit Hyper- 


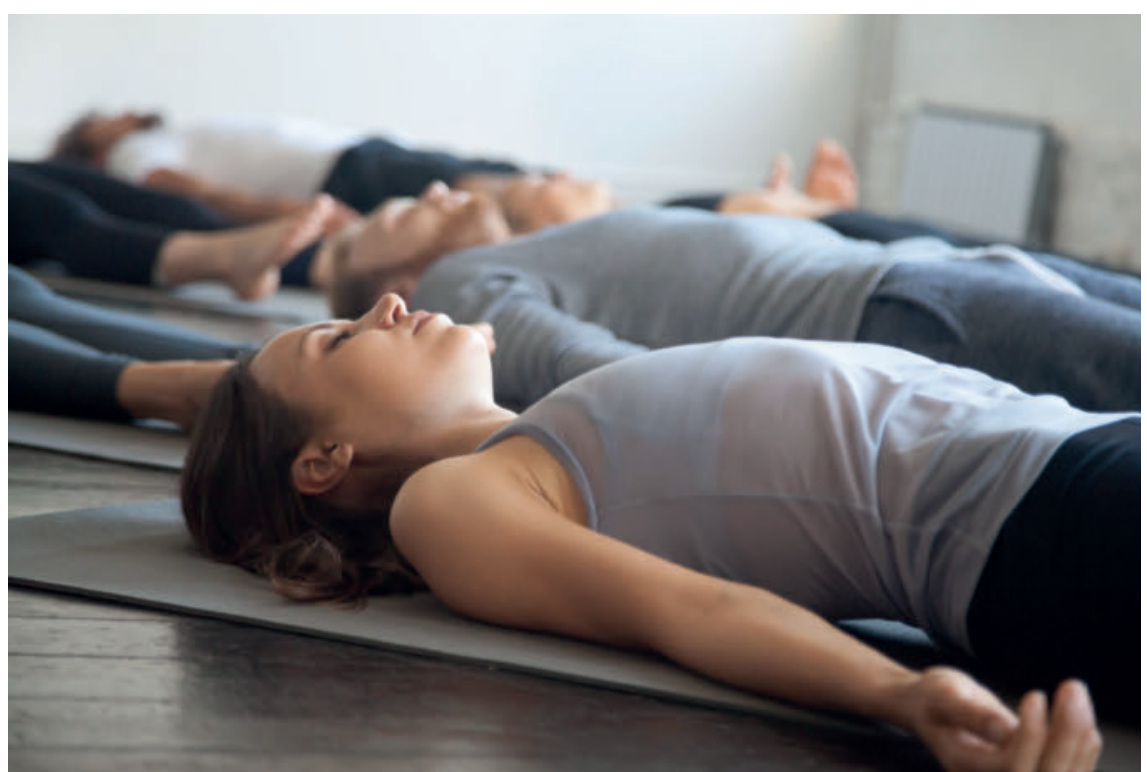

Abb. 2 Die blutdrucksenkende Wirkung von Entspannungsverfahren ist in Studien und Metaanalysen belegt. (C) fizkes / Adobe Stock; nachgestellte Situation

tonie, Stadium I-II, hierdurch eine Medikamentenreduktion möglich, $50 \%$ sind im weiteren Verlauf ohne Medikation normotensiv.

\section{Hydrotherapie, Balneotherapie}

Bei serieller Therapie mit hydrotherapeutischen Anwendungen kommt es zur physikalischen Vasodilatation (Warmreize) und reaktiver Adaptation (Kaltreize) mit Einleitung einer Blutdrucknormalisierung. Die serielle, insbesondere häusliche Hydrotherapie ist damit als adjuvante, nicht pharmakologische Therapie sehr geeignet.

Blutdrucksenkende Akuteffekte durch passiv oder reaktiv wärmende vasodilatierende Anwendungen sind insbesondere dokumentiert für

- feuchtkalte Brust- und Leibwickel (45 Minuten)

- wechselwarme Teilgüsse

- ansteigende Teilbäder, insbesondere Armbäder

- Senfmehl- bzw. Ingwer-Fußbäder bei Neigung zu kalten Füßen und oberer Fülle

- Vollbäder, gegebenenfalls mit entspannungsfördernden Zusätzen (Lavendel, Melisse)

- Kohlendioxidbäder

Güsse und Bäder sind nach der Klinikbehandlung möglichst 2-mal täglich als Selbstanwendung zu nutzen. Bei Kohlendioxidbädern fällt der periphere Widerstand sofort durch Weitstellung der Kapillargefäße, damit sinkt der systolische Blutdruck. Durch thermorezeptorisch inhibierte Kältegegenregulation entsteht eine leichte Hypothermie, zusätzlich ein gewisser sedierender Effekt. Bei serieller Anwendung treten vermutlich durch adaptive Prozesse nachhaltige

\section{Phytotherapie}

Insgesamt sind die antihypertensiven Effekte der Phytotherapie als gering einzuschätzen:

Weißdornextrakte aus Blättern und Blüten sind marginal blutdrucksenkend und v.a. bei Hypertonie mit diastolischer oder systolischer Myokardinsuffizienz sinnvoll.

Knoblauchpulver verbessert v.a. die Gefäßelastizität (z.B. Sapec ${ }^{\circledR}, 3 \times 1$ Drg.). Der blutdrucksenkende Effekt wurde in 20 Studien untersucht und erbrachte im gewichteten Mittel eine Absenkung von $5 \mathrm{mmHg}$ systolisch, bzw. $3 \mathrm{mmHg}$ diastolisch [5].

Melissentee kann zur grundsätzlichen Stressreduktion bei Bluthochdruck beitragen.

Hoffnungsvoll erscheint die noch recht junge Forschung zu Hibiskustee, der mit meist 3 Tassen / $d$ in mittlerweile 8 randomisierten Studien und einem systematischen Review Blutdrucksenkungen im gewichteten Mittel von $8 \mathrm{mmHg}$ systolisch, bzw. $4 \mathrm{mmHg}$ diastolisch erbrachte [6].

\section{Ausleitende Verfahren Aderlass}

Der Aderlass eignet sich als wirksame ergänzende Therapie bei Bluthochdruck. Die Wirksamkeit wurde inzwischen in einer

\section{Der Aderlass eignet sich als wirksame ergänzende} Therapie bei Bluthochdruck.

Blutdrucksenkungen ein. Kohlendioxidbäder sind in Form serieller Anwendungen 2bis 6-mal wöchentlich über 3-6 Wochen durchzuführen. Allerdings werden sie aufgrund des hohen apparativen Aufwands und nicht einmal kostendeckender Abrechenbarkeit nach GOÄ professionell immer seltener angeboten, die Eigenanwendung mit sog. Kohlensäurebädern in Pulverform ist weiterhin möglich.

Eine Thermowärmetherapie kann bei Patienten mit peripherer Vasokonstriktion günstige Wirkungen entfalten. Zum Einsatz kommen Vollbäder und Saunabäder, jeweils mit moderaten Temperaturen. Bluthochdruckpatienten dürfen kein kaltes Tauchbad nach dem Saunagang vornehmen (Blutdrucksteigerung!). Alternativ kann auch die systemische Infrarot-A-Hyperthermie probatorisch eingesetzt werden. randomisiert-kontrollierten Studie belegt, alternativ kann Patienten die Blutspende empfohlen werden (s. Beitrag S. 56). Traditionell werden in der Naturheilkunde kleinere Aderlässe mit Entnahmemengen von $<250 \mathrm{ml}$ wiederholt entnommen. In bisherigen wissenschaftlichen Studien haben sich größere Aderlässe $(350-500 \mathrm{ml})$ in etwa 4-8-wöchigen Abständen als deutlich blutdrucksenkend erwiesen. Insbesondere bei entsprechender Konstitution (Fülle, „Plethora“) sowie höherem Hämatokrit (> $45 \%$ ) oder erhöhtem Ferrtin-Spiegel (>150) erscheint ein Aderlass sinnvoll.

\section{Schröpfen}

Blutiges Schröpfen ist bei der sog. „heißen Gelose“ in beschriebenen „Hypertoniezonen“ (z.B. nach Abele) ein mögliches additives Verfahren. Beim Leeretyp („weißer“ 
Bluthochdruck) sollten trockenes Schröpfen und Anregung der Wärmebildung durch Hydrotherapie erfolgen. Traditionelle Hypertoniezonen sind oberhalb des Proc. spinosus L5/S1 oder auch die „Nierenzone“ L1/ L2 paravertebral sowie im Bereich des 7. HWK („Hormonbuckel“). Die Existenz von sog. Hypertonie-Reflexzonen ist allerdings bislang nicht gesichert. Eine spezifische Wirkung ist bislang nicht belegt.

\section{Massagen}

Massagen verbessern allgemein die periphere Durchblutung und senken damit den peripheren Widerstand. Zudem sind akut antihypertensive Effekte mittels der psychovegetativen Entlastung, Beseitigung muskulärer Verspannungen und reaktiven Diurese erzielbar. Allerdings ist nicht bekannt, ob hierdurch auch Langzeiteffekte möglich sind. Bei sog. fülligen Bindegewebszonen im Bereich der Hypertoniezonen kann probatorisch ein Therapieversuch z. B. mit Bindegewebsmassage erfolgen.

\section{Heliotherapie}

Auch für die UVB-Heliotherapie ist eine blutdrucksenkende und günstige kardiovaskuläre Wirkung belegt. Heliotherapeutische serielle Behandlungen mittels spezieller UVB-Liegen in suberythematöser Dosis können unter Beachtung des Hauttyps insbesondere in den Wintermonaten additiv eingesetzt werden.

\section{Akupunktur}

Die antihypertensive Wirksamkeit der Akupunktur ist durch Studien inzwischen belegt, allerdings sind die antihypertensiven Effekte moderat und bleiben nach Absetzen der Therapie nicht bestehen, sodass eine fortdauernde Therapie notwendig wäre. Sinnvoll kann aber die Akupunktur als „bridging“ und als ergänzende vegetativ umstimmende Therapie in multimodalen Therapieprogrammen zum Einsatz kommen.

Lesen Sie im Beitrag ab S. 18 welche komplementären Therapieoptionen für die koronare Herzkrankheit und die Herzinsuffizienz belegt sind.
Interessenkonflikt: Die Autoren erklären, dass keine wirtschaftlichen oder persönlichen Verbindungen bestehen.

\section{Online zu finden unter}

http://dx.doi.org/10.1055/a-0724-5808

\section{Literatur}

\section{Übersichten zum Thema}

$\overline{1}$ Michalsen A. Therapie der Herz-Kreislauferkrankungen. In: Kraft K, Stange R, Hrsg. Lehrbuch Naturheilverfahren. Stuttgart: Hippokrates; 2010

$\overline{2}$ Budde T, Michalsen A. Koronare Herzerkrankung, Bluthochdruck. In: Dobos G, Michalsen A, Deuse U, Hrsg. Chronische Krankheiten integrativ. München: Elsevier, Urban und Fischer; 2006

$\overline{3}$ Paul A, Michalsen A. Natürlich herzgesund. Essen: KVC Verlag; 2008

$\overline{4}$ Huber R, Michalsen A, Hrsg. Checkliste Komplementärmedizin. Stuttgart: Haug; 2014

\section{Zitierte Literatur}

$\overline{5}$ Ried K. Garlic lowers blood pressure in hypertensive individuals, regulates serum cholesterol, and stimulates immunity: An updated meta-analysis and review. J Nutr 2016; 146 (2): 389S-396S. doi: $10.3945 / j n .114 .202192$

$\overline{6}$ Serban C, Sahebkar A, Ursoniu S et al. Effect of sour tea (Hibiscus sabdariffa L.) on arterial hypertension: a systematic review and meta-analysis of randomized controlled trials. J Hypertens 2015; 33 (6): 1119-1127. doi: $10.1097 \mid$ HJH.0000000000000585

Weitere Literatur beim Verfasser.

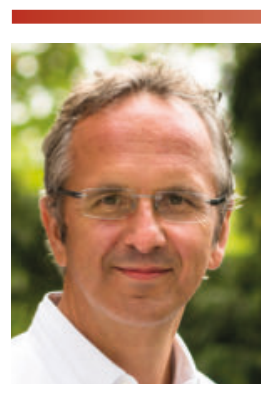

Prof. Dr. med. Andreas Michalsen

Stiftungsprofessur für klinische Naturheilkunde Charité - Universitätsmedizin Berlin

Abteilung Naturheilkunde

Immanuel Krankenhaus Berlin, Standort BerlinWannsee

Königstr. 63, 14109 Berlin

a.michalsen@immanuel.de

Andreas Michalsen ist Facharzt für Innere Medizin mit den Zusatzbezeichnungen Naturheilverfahren, Ernährungsmedizin, Physikalische Medizin und Balneologie. Nach Stationen in Berlin und Bad Elster war er von 1999-2008 leitender Oberarzt der Abteilung Innere Medizin V, Naturheilkunde und Integrative Medizin der Kliniken Essen-Mitte. Seit 2009 Inhaber der Professur für klinische Naturheilkunde, Charité - Universitätsmedizin und Immanuel Krankenhaus Berlin sowie Vorstandsvorsitzender der Carstens-Stiftung.

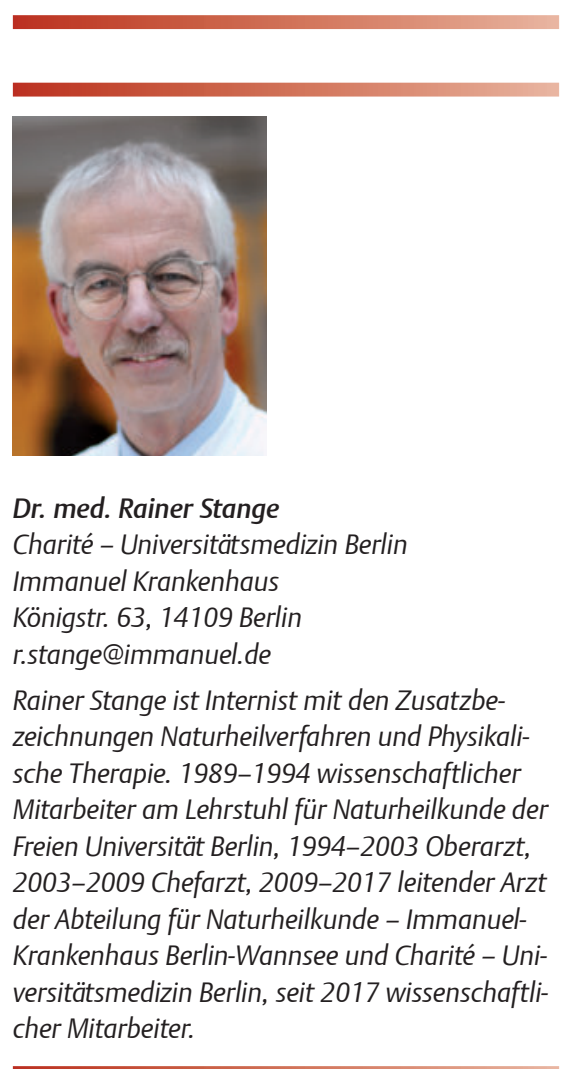

